\begin{tabular}{|c|l|}
\hline Title & $\begin{array}{l}\text { FIP1L1 presence in FIP1L1-RA RA or FIP1L1-PDGFRA differentially contributes to the pathogenesis of distinct types } \\
\text { of leukemia }\end{array}$ \\
\hline Author(s) & $\begin{array}{l}\text { Iwasaki, Junko; Kondo, Takeshi; Darmanin, Stephanie; Ibata, Makoto; Onozawa, Masahiro; Hashimoto, Daigo; } \\
\text { Sakamoto, Naoya; Teshima, Takanori }\end{array}$ \\
\hline Citation & $\begin{array}{l}\text { A nnals of Hematology, 93(9), 1473-1481 } \\
\text { https://doi.org/10.1007/300277-014 2085-1 }\end{array}$ \\
\hline Issue Date & 201409 \\
\hline Doc URL & http://hdl.handle.net/2115/59854 \\
\hline Rights & The final publication is available at link.springer.com \\
\hline Type & article (author version) \\
\hline File Information & A OHE-D-13 00868.pdf \\
\hline
\end{tabular}

Instructions for use 


\section{Annals of Hematology \\ FIP1L1 presence in FIP1L1-RARA or FIP1L1-PDGFRA differentially contributes to the pathogenesis of distinct types of leukemia --Manuscript Draft--}

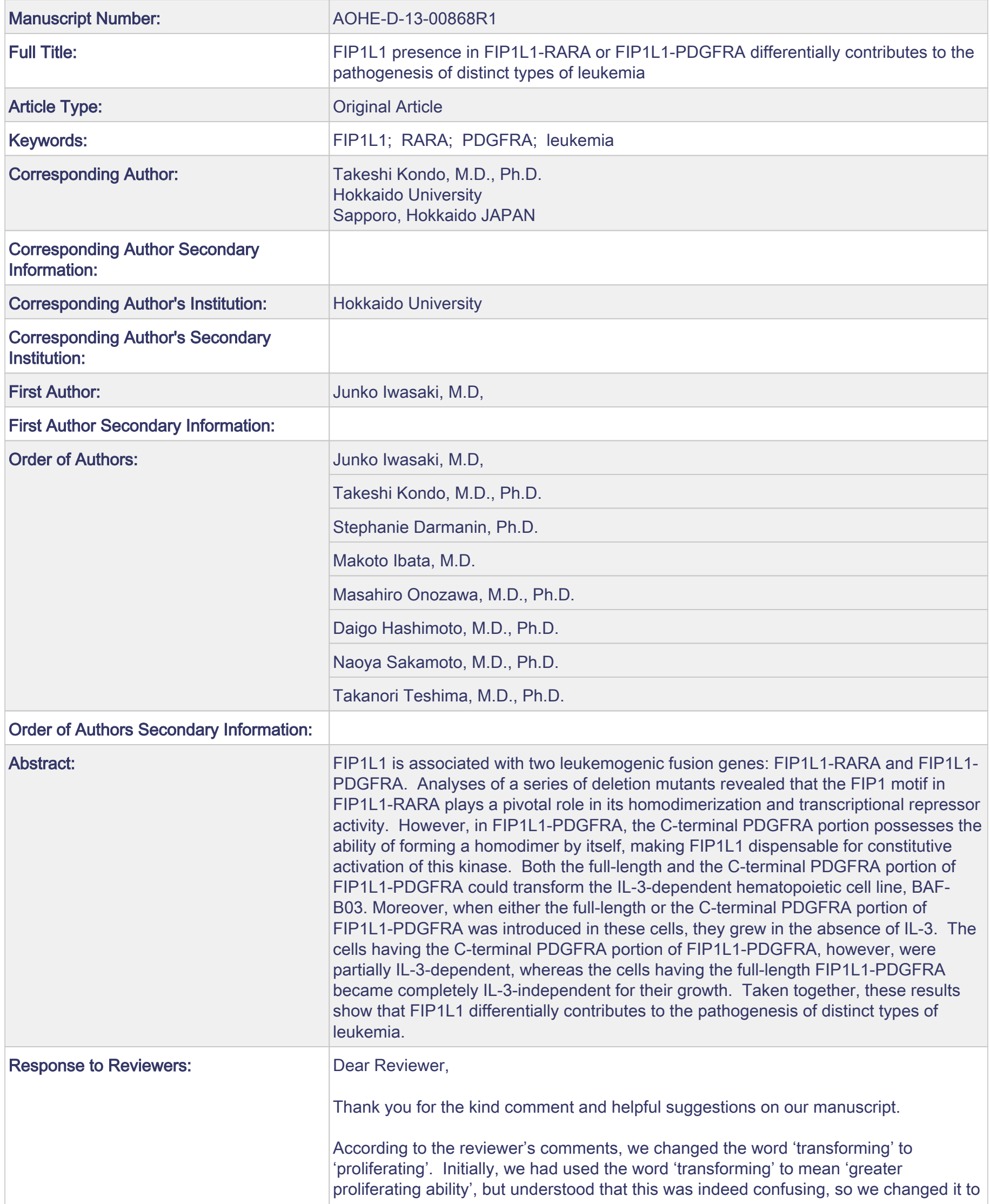


'proliferating'.

In addition, as per the reviewer's suggestions, we examined imatinib sensitivity of FIP1L1-PDGFRA-FL, FIP1L1-PDGFRA-dFIP1/Ex9 and PDGFRA-C. However, because we did not see any differences in responses to imatinib, we are not adding any data obtained from this experiment to the manuscript. We tried to characterize the differences of FIP1L1-PDGFRA-FL, FIP1L1-PDGFRA-dFIP1/Ex9 and PDGFRA-C. In Figure 5, we show the intracellular localization of FIP1L1-PDGFRA-FL, FIP1L1PDGFRA-dFIP1/Ex9 and PDGFRA-C, which is explained in the 'Discussion' section.

We thank you in advance for reviewing our manuscript.

Yours sincerely,

Takeshi Kondo, MD, PhD

Department of Hematology and Oncology

Hokkaido University Graduate School of Medicine 
FIP1L1 presence in FIP1L1-RARA or FIP1L1-PDGFRA differentially contributes to the pathogenesis of distinct types of leukemia

Junko Iwasaki ${ }^{1)}$, Takeshi Kondo1), Stephanie Darmanin'2)3), Makoto Ibata1), Masahiro Onozawa ${ }^{1)}$, Daigo Hashimoto' ${ }^{1)}$, Naoya Sakamoto ${ }^{2)}$ and Takanori Teshima $^{1)}$

1) Department of Hematology, Hokkaido University Graduate School of Medicine

2) Department of Gastroenterology and Hepatology, Hokkaido University Graduate School of Medicine

3) Center for Hematology and Regenerative Medicine, Department of Medicine, Karolinska University Hospital

Running title: FIP1L1 in leukemogenesis

Correspondence: Takeshi Kondo, Department of Hematology, Hokkaido University Graduate School of Medicine, Kita 15, Nishi 7, Kita-ku, Sapporo, Hokkaido 060-8638, Japan; e-mail: t-kondoh@med.hokudai.ac.jp 


\begin{abstract}
FIP1L1 is associated with two leukemogenic fusion genes: FIP1L1-RARA and FIP1L1-PDGFRA. Analyses of a series of deletion mutants revealed that the FIP1 motif in FIP1L1-RARA plays a pivotal role in its homodimerization and transcriptional repressor activity. However, in FIP1L1-PDGFRA, the C-terminal PDGFRA portion possesses the ability of forming a homodimer by itself, making FIP1L1 dispensable for constitutive activation of this kinase. Both the full-length and the C-terminal PDGFRA portion of FIP1L1-PDGFRA could transform the IL-3-dependent hematopoietic cell line, BAF-B03. Moreover, when either the full-length or the C-terminal PDGFRA portion of FIP1L1-PDGFRA was introduced in these cells, they grew in the absence of IL-3. The cells having the C-terminal PDGFRA portion of FIP1L1-PDGFRA, however, were partially IL-3-dependent, whereas the cells having the full-length FIP1L1-PDGFRA became completely IL-3-independent for their growth. Taken together, these results show that FIP1L1 differentially contributes to the pathogenesis of distinct types of leukemia.
\end{abstract}

Key Words:

FIP1L1

RARA

PDGFRA

Leukemia 


\section{Introduction}

FIP1L1 (FIP1-like 1) is a human homologue of the Saccharomyces cerevisiae FIP1, which was initially isolated as a fusion gene with platelet-derived growth factor receptor alpha (PDGFRA) from patients with idiopathic hypereosinophilic syndrome/chronic eosinophilic leukemia (HES/CEL) [1, 2]; FIP1L1-PDGFRA-positive HES/CEL being an independent clinical entity [3]. In addition to the FIP1L1-PDGFRA fusion gene, FIP1L1 has also been reported to form a fusion gene with retinoic acid receptor alpha (RARA), FIP1L1-RARA, in cases of juvenile myelomonocytic leukemia and acute promyelocytic leukemia [4-6]. FIP1L1 is therefore known to be associated with, at least, two distinct fusion genes that contribute to the development of leukemia.

In FIP1L1-RARA, the FIP1L1 portion is necessary for both homodimer formation, as well as the repression of retinoic acid-responsive transcriptional activity [5]. Interestingly, although FIP1L1 is reported to possess the ability to form a homodimer, it does not have a known protein-protein interaction domain.

FIP1L1-PDGFRA is a constitutively active tyrosine kinase; this kinase activity being crucial for transforming hematopoietic cells. A previous report indicated that disruption of the juxtamembrane domain of PDGFRA is critical for constitutive activation of the kinase, whereas the FIP1L1 portion is dispensable [7]. Recently, it has been found that the FIP1L1 portion is also necessary for activating STAT5 and PKB/Akt in human hematopoietic progenitor cells [8].

At the moment, little is known about the role of FIP1L1 in these two different fusion genes associated with leukemogenesis. In this study, we aimed to investigate the pathological roles of FIP1L1 in the oncogenic potential of these two fusion genes.

\section{Materials and methods}

\section{Plasmid construction}


FIP1L1-RARA cDNA was originally isolated from a patient with acute promyelocytic leukemia [5]. FIP1L1-RARA is composed of 426 amino acids (aa) from the N-terminus of FIP1L1 and 403 aa from the C-terminus of RARA. FLAG-tagged and T7-tagged expression vectors of FIP1L1-RARA, pFLAG-FIP1L1-RARA and pCGT-FIP1L1-RARA respectively, were constructed as previously described [5]. A series of deletion mutants of FIP1L1-RARA was generated by polymerase chain reaction (PCR) (Figure 1a). FIP1L1-N152, FIP1L1-N242 and FIP1L1-N339 constructs are composed of the 152, 242 and $339 \mathrm{~N}$-terminal aa of FIP1L1, respectively. N-terminal deletion mutants of FIP1L1-RARA lacking 134 and 235 aa were named FIP1L1-RARA-dN134 and FIP1L1-RARA-dN235, respectively. Internal deletion mutants of FIP1L1-RARA lacking amino acid regions 153 to 212, 213 to 235 and 153 to 235, were named FIP1L1-RARA-dFIP1, FIP1L1-RARA-dEx9 and FIP1L1-RARA-dFIP1/Ex9, respectively (Figure 1b). These mutants were cloned into FLAG-tagged expression vectors. PDGFRA cDNA was cloned from total HeLa cell RNA by reverse transcription (RT)-PCR. FIP1L1-PDGFRA cDNA was constructed by fusing the 5'-portion of FIP1L1 cDNA to the 3'-portion of PDGFRA cDNA. The FIP1L1-PDGFRA cDNA obtained encodes $339 \mathrm{~N}$-terminal aa of FIP1L1 and 480 C-terminal aa of PDGFRA. Although there are several variants of the FIP1L1-PDGFRA cDNA, this construct represents one of the most common sequences of the FIP1L1-PDGFRA fusion gene [1]. Full-length FIP1L1-PDGFRA cDNA was cloned into a FLAG-tagged expression vector or a T7-tagged expression vector, and named pFLAG-FIP1L1-PDGFRA-FL and pCGT-FIP1L1-PDGFRA-FL respectively. An internal deletion mutant of FIP1L1-PDGFRA was also constructed. This mutant was named FIP1L1-PDGFRA-dFIP1/Ex9, and lacked aa regions 153 to 235. A mutant with only the C-terminal portion of PDGFRA, lacking the FIP1L1 portion, was also constructed and named PDGFRA-C (Figure 1c). A kinase-dead mutant, FIP1L1-PDGFRA-KD, was generated by introducing a lysine-to-arginine mutation at amino acid position 627 of PDGFRA, by means of site-directed mutagenesis [9]. To generate retroviral vectors, cDNAs of T7-tagged FIP1L1-PDGFRA-FL, FIP1L1-PDGFRA-dFIP1/Ex9 and 
PDGFRA-C were cloned into pBabe-Puro.

\section{Cell culture}

HEK293 cells were cultured in Dulbecco's modified Eagle's medium supplemented with $10 \%$ fetal bovine serum. BAF-B03 cells were cultured in RPMI-1640 medium supplemented with $10 \%$ fetal bovine serum and 1 $\mathrm{ng} / \mathrm{ml}$ murine IL-3 (Medical \& Biological Laboratories). To establish BAF-B03 cells expressing FIP1L1-PDGFRA, we used the retrovirus packaging kit Eco (TaKaRa). BAF-B03 cells were infected with a retrovirus harboring full-length or deletion mutants of FIP1L1-PDGFRA and selected with puromycin. BAF-B03-derived cells were cultured in the presence or absence of IL-3, to examine IL-3-independent growth.

\section{Transfection, immunoblotting, immunostaining and luciferase assays}

For transfection experiments, HEK293 cells were grown in a 6 - $\mathrm{cm}$ dish and transfected with expression vectors using Lipofectamine 2000. Immunoprecipitation, immunoblotting and immunostaining analyses were carried out as described previously [10]. Anti-FLAG M2 antibody, anti-T7 tag and anti-phosphotyrosine (PY20) antibody were purchased from Sigma, Medical \& Biological Laboratories and Wako Pure Chemical Industries, respectively. For immunostaining, Alexa Fluor ${ }^{\circledR} 488$ goat anti-mouse IgG was purchased from Invitrogen. For luciferase analysis, the effectors used were expression vectors for pFLAG-FIP1L1-RARA, pFLAG-FIP1L1-RARA-dFIP1, pFLAG-FIP1L1-RARA-dEx9 and pFLAG-FIP1L1-RARA-dFIP1/Ex9, and the reporter gene was the retinoic acid-responsive luciferase vector. Luciferase activities were analyzed as previously described [5].

\section{Results}

The region surrounding the FIP1 motif is a homodimerization domain Initially, we analyzed the pathological role of FIP1L1 in FIP1L1-RARA. 
FIP1L1 is a human homologue of the Saccharomyces cerevisiae polyadenylation factor Fip1p [11], and it is an integral subunit for cleavage and polyadenylation specificity factor (CPSF) in the stimulation of poly(A) polymerase. FIP1L1 is conserved among various species and the FIP1 motif is a hallmark of FIP1L1. Because this 40 aa-long FIP1 motif is required to form the CPSF complex, we hypothesized that it might play a significant role in protein-protein interactions.

Because FIP1L1-RARA homodimerization is required for suppressing retinoic acid-dependent transcriptional activity, we tried to map the region of FIP1L1 that is necessary for homodimerization. In the cDNA of FIP1L1-RARA, exon 15 of FIP1L1 is fused to exon 3 of RARA, in which the FIP1L1 portion is translated into 428 aa. We initially generated a series of either $\mathrm{N}$-terminal or $\mathrm{C}$-terminal deletion mutants of FIP1L1-RARA in FLAG-tagged expression vectors (Figure 1a). These mutants were co-expressed with T7-tagged full-length FIP1L1-RARA, and their association was analyzed. The first $152 \mathrm{~N}$-terminal aa of FIP1L1 (FIP1L1-N152) showed no ability to associate with full-length FIP1L1-RARA. However, both FIP1L1-N242 and FIP1L1-N339, representing the first 242 and 339 $\mathrm{N}$-terminal aa of FIP1L1, could associate with FIP1L1-RARA. In agreement with these findings, while FIP1L1-RARA-dN134, the N-terminal deletion mutant of FIP1L1-RARA lacking the first $134 \mathrm{~N}$-terminal aa, retained the ability to dimerize, FIP1L1-RARA-dN235, the N-terminal deletion mutant of FIP1L1-RARA lacking the first $235 \mathrm{~N}$-terminal aa, could not form a dimer. Taken together, these results show that the FIP1L1 region between aa 135 and 236, which includes the FIP1 motif, is essential for homodimerization (Figure 2a). Therefore, we next generated three internal deletion mutants in FLAG-tagged expression vectors, lacking amino acid regions 153 to 212, 213 to 235, and 153 to 235 of FIP1L1-RARA, which we named FIP1L1-RARA-dFIP1, -dEx9 and -dFIP1/Ex9, respectively (Figure 1b). As shown in Figure $2 \mathrm{~b}$, the association between full-length FIP1L1-RARA and FIP1L1-RARA-dFIP1 became much weaker than that between the wild-type proteins, but was still detected (Figure 2b, lane 4). FIP1L1-RARA-dEx9 efficiently associated with full-length FIP1L1-RARA 
(Figure 2b, lane 5), while FIP1L1-RARA-dFIP1/Ex9 showed almost no association with full-length FIP1L1-RARA (Figure 2b, lane 6), comparable to the association of FIP1L1-RARA to the -dFIP1 mutant. From these results, we therefore surmise that the homodimerization of FIP1L1-RARA is mediated mainly through its FIP1 motif.

We then moved on to examine the effect of these mutants on retinoic-acid responsive transcription. Luciferase assays revealed that full-length FIP1L1-RARA repressed retinoic acid-dependent transcriptional activity, but for the deletion mutants of FIP1L1-RARA this repressive activity correlated with their ability to form a homodimer. FIP1L1-RARA-dEx9 efficiently repressed luciferase activity, but FIP1L1-RARA-dFIP1 and -dFIP1/Ex9 showed no repressive activity (Figure 2c). Collectively, these findings show that the FIP1 motif plays a pivotal role in both homodimer formation and transcriptional repressor activity of FIP1L1-RARA, consistent with the initial hypothesis that the FIP1 motif is a protein-protein interaction domain.

The C-terminal portion of PDGFRA, by itself, has the ability to homodimerize

Because FIP1L1 is also associated with eosinophilic leukemia, we also examined its pathological role in FIP1L1-PDGFRA. Constitutive activation of FIP1L1-PDGFRA is reported to be dependent on disruption of the juxtamembrane domain of PDGFRA [7]; however, because our results clearly indicate that FIP1L1 possesses a homodimerization domain, we next examined whether FIP1L1 also plays a role in FIP1L1-PDGFRA homodimerization.

Several variants of FIP1L1 exist in FIP1L1-PDGFRA, depending on the breakpoints in the genome. Interestingly, however, all these variants usually contain the FIP1L1 sequence spanning aa 152 to 236, which is its homodimerization domain. We used a FIP1L1-PDGFRA cDNA, containing exons 1 to 13 of FIP1L1 [1, 12]. Moreover, we generated a deletion mutant of FIP1L1-PDGFRA, lacking aa 153 to 235, which we named FIP1L1-PDGFRA-dFIP1/Ex9 (Figure 1c). We then cloned these cDNAs into 
FLAG-tagged or T7-tagged expression vectors, which we named FIP1L1-PDGFRA-FL or FIP1L1-PDGFRA-dFIP1/Ex9, respectively. Transient transfection experiments, however, revealed that this mutant does not inhibit homodimerization; indeed, FIP1L1-PDGFRA-dFIP1/Ex9 forms a homodimer as efficiently as FIP1L1-PDGFRA-FL (Figure 3a), which clearly means that FIP1L1-PDGFRA homodimerization is not mediated by the FIP1 motif, and that, therefore, FIP1L1-PDGFRA forms a homodimer in a different manner from FIP1L1-RARA.

This result prompted us to check whether the C-terminal PDGFRA portion by itself has the ability to form a homodimer. We generated FLAG-tagged or T7-tagged expression vectors of the C-terminal PDGFRA portion of FIP1L1-PDGFRA, which we named PDGFRA-C (Figure 1c), and conducted transient transfection experiments. As shown in Figure 3b, the association of PDGFRA-C with itself was as good as its association to FIP1L1-PDGFRA-FL.

Additionally, we wanted to verify that FIP1L1-PDGFRA-FL, FIP1L1-PDGFRA-dFIP1/Ex9 and PDGFRA-C are indeed constitutively active kinases. Flag-tagged expression vectors of FIP1L1-PDGFRA-FL, FIP1L1-PDGFRA-dFIP1/Ex9, PDGFRA-C or kinase-inactive FIP1L1-PDGFRA-FL were transfected into 293 cells, and their auto-phosphorylation status was analyzed. As shown Figure 3c, FIP1L1-PDGFRA-FL, FIP1L1-PDGFRA-dFIP1/Ex9 and PDGFRA-C showed kinase activity, with phosphorylation at tyrosine residues, while the kinase-dead mutant of FIP1L1-PDGFRA-FL was not phosphorylated. Thus, this phosphorylation was dependent on the kinase activity of FIP1L1-PDGFRA and PDGFRA-C.

Altogether, these results indicate that the C-terminal portion of PDGFRA, by itself, possesses the ability to homodimerize and is a constitutively active kinase. These results are consistent with a previous report, describing that the FIP1L1 portion is dispensable for activation of FIP1L1-PDGFRA for its kinase activity [7].

FIP1L1-PDGFRA-expressing cells show greater proliferating activity than 
those expressing PDGFRA-C

To analyze the pathological role of the FIP1L1 portion of FIP1L1-PDGFRA, we established BAF/B03-derived stable transformants expressing FIP1L1-PDGFRA-FL， FIP1L1-PDGFRA-dFIP1/Ex9 or PDGFRA-C, respectively (Figure 4a). Parental BAF-B03 cells are an IL-3-dependent pro-B cell line, which becomes IL-3-independent upon the introduction of a kinase-active PDGFRA [7]. In accordance with the previous results demonstrating the ability of PDGFRA to homodimerize and to be an active kinase, these three transformants grew without IL-3, while the empty vector-expressing cells manifested IL-3-dependent growth (Figure 4b to 4e). While establishing the stable transformants, we noticed that the cells expressing PDGFRA-C grow slower in the absence of IL-3 than in its presence. To confirm this phenomenon, we examined the growth characteristics of these transformants with or without IL-3. Here, we used cells at an early passage, to avoid observing any effects of clonal variation. Cells $\left(1 \times 10^{3}\right)$ in exponential growth phase were prepared and cultured in the presence or absence of IL-3, and their numbers were counted in a time-course experiment. As shown in Figure $4 \mathrm{c}$ and $4 \mathrm{~d}$, the cells expressing either FIP1L1-PDGFRA-FL or FIP1L1-PDGFRA-dFIP1/Ex9 grew steadily, irrespective of the presence of IL-3. On the other hand, the cells expressing PDGFRA-C manifested higher growth in the presence of IL-3 than in its absence (Figure 4e). This result indicates that the stable transformant expressing PDGFRA-C remains partially IL-3-dependent, while the stable transformants expressing FIP1L1-PDGFRA-FL or FIP1L1-PDGFRA-dFIP1/Ex9 become completely IL-3-independent. These results suggest that FIP1L1 is necessary for the higher proliferating activity of FIP1L1-PDGFRA-FL, when compared to PDGFRA-C.

\section{FIP1L1 directs FIP1L1-PDGFRA primarily to the nucleus}

To elucidate the difference in proliferating activity of FIP1L1-PDGFRA-FL, FIP1L1-PDGFRA-dFIP1/Ex9 and PDGFRA-C, we assumed that the intracellular behavior of PDGFRA-C differs from that of FIP1L1-PDGFRA-FL and FIP1L1-PDGFRA-dFIP1/Ex9. We therefore tried 
to examine the intracellular localization of these molecules. T7-tagged expression vectors of FIP1L1-PDGFRA-FL, FIP1L1-PDGFRA-dFIP1/Ex9 and PDGFRA-C were transfected into 293 cells, and the expressed proteins were immunostained by anti-T7 antibody. As shown in Figure 5, both FIP1L1-PDGFRA-FL and FIP1L1-PDGFRA-dFIP1/Ex9 are located primarily in the nucleus (Figures 5b and 5c), while PDGFRA-C exists mainly in the cytoplasm (Figure 5d). This result indicates that FIP1L1 directs FIP1L1-PDGFRA primarily to the nucleus.

\section{Discussion}

Here, we analyzed the pathological roles of FIP1L1 in two FIP1L1-associated fusion genes for leukemogenesis: FIP1L1-RARA and FIP1L1-PDGFRA. FIP1L1 is a human homologue of the Saccharomyces cerevisiae polyadenilation factor Fip1p (11). It is reported that FIP1L1 is an integral subunit for cleavage and polyadenylation specificity factor (CPSF) for stimulation of poly(A) polymerase. FIP1L1 is conserved among various species and the FIP1 motif is a hallmark for FIP1L1. The FIP1 motif is a short motif, about 40 amino acids long, necessary to form the CPSF complex. We therefore hypothesized that the FIP1 motif plays a role in protein-protein interaction. FIP1L1-RARA functions as a transcriptional repressor in retinoic acid-dependent transcription, and homodimerization of FIP1L1-RARA is crucial for this activity (5). Here, we tried to map the region of FIP1L1-RARA essential for homodimerization. By analyzing a series of deletion mutants of FIP1L1-RARA, we confirmed that the FIP1 motif plays a pivotal role in homodimerization. This result is consistent with the notion that the FIP1 motif is a protein-protein interaction domain.

Since FIP1L1 is also associated with eosinophilic leukemia, we examined the pathological role of the FIP1L1 portion in FIP1L1-PDGFRA. The C-terminal portion of PDGFRA in FIP1L1-PDGFRA, by itself, had the ability to transform BAF-B03 cells. We attribute this finding to the fact that the C-terminal portion of PDGFRA itself has the ability to form a homodimer and the kinase activity of PDGFRA-C should be constitutively active. We 
also established FDC-P2-derived cell lines. FDC-P2 is a murine myeloid cell line that also manifests IL-3-dependent growth [13]. Similarly to the results obtained for BAF-B03-derived cells, the FDC-P2-derived cells expressing FIP1L1-PDGFRA-FL or FIP1L1-PDGFRA-dFIP1/Ex9 became completely IL-3-independent, while the stable transformant expressing PDGFRA-C was partially IL-3-dependent for growth (data not shown).

These results were consistent with previous reports, stating that the FIP1L1 portion is dispensable for the activation of FIP1L1-PDGFRA kinase activity [7]. The FIP1L1 portion is, however, a contributing factor to the higher proliferating activity, induced by FIP1L1-PDGFRA. This result is also consistent with a previous study, which shows that the FIP1L1 portion is necessary to activate STAT5 and the PKB/Akt pathway in human hematopoietic progenitor cells [8].

Moreover, our immunostaining results indicate that FIP1L1-PDGFRA, both -FL and -dFIP1/Ex9, is located primarily in the nucleus, while PDGFRA-C is mainly distributed within the cytoplasm. The FIP1 motif seems to be unnecessary for the nuclear retention of FIP1L1-PDGFRA, and this phenomenon is parallel to the higher proliferating activity of cells expressing FIP1L1-PDGFRA compared to those expressing PDGFRA-C.

It should also be considered that the FIP1L1 portion associates with other molecules and it could be that these molecules are involved in the nuclear retention or the higher proliferating activity of FIP1L1-PDGFRA. The $\mathrm{N}$-terminal acidic region of FIP1L1 is necessary for CPSF to associate with poly(A) polymerase and this region is retained in FIP1L1-PDGFRA-dFIP1/Ex9 (7). It would be interesting to analyze the molecules that associate with the FIP1L1 portion of FIP1L1-PDGFRA, in an attempt to elucidate the oncogenic pathways of FIP1L1-PDGFRA.

In conclusion, the FIP1L1 portions of both FIP1L1-RARA and FIP1L1-PDGFRA, in a distinct manner, contribute to the pathogenesis of different types of leukemias.

\section{Acknowledgements}


1

J.I. designed and performed experiments; T.K. designed experiments and wrote the manuscript; M.I. and M.O. performed experiments; D.H. analyzed data; S.D., N.S. and T.T. corrected and completed the manuscript. The authors declare that they have no conflict of interest. 
References

1. Cools J, DeAngelo DJ, Gotlib J, Stover EH, Legare RD, Cortes J, Kutok J, Clark J, Galinsky I, Griffin JD, Cross NC, Tefferi A, Malone J, Alam R, Schrier SL, Schmid J, Rose M, Vandenberghe P, Verhoef G, Boogaerts M, Wlodarska I, Kantarjian H, Marynen P, Coutre SE, Stone R, Gilliland DG (2003) A tyrosine kinase created by fusion of the PDGFRA and FIP1L1 genes as a therapeutic target of imatinib in idiopathic hypereosinophilic syndrome. N. Engl. J. Med. 348: 1201-1214.

2. Gotlib J, Cools J. (2008) Five years since the discovery of FIP1L1-PDGFRA: what we have learned about the fusion and other molecularly defined eosinophilias. Leukemia 22: 1999-2010.

3. Lierman E, Cools J (2009) Recent breakthroughs in the understanding and management of chronic eosinophilic leukemia. Expert Rev. Anticancer Ther. 9: 91295-91304.

4. Buijs A, Bruin M (2007) Fusion of FIP1L1 and RARA as a result of a novel $\mathrm{t}(4 ; 17)(\mathrm{q} 12 ; \mathrm{q} 21)$ in a case of juvenile myelomonocytic leukemia. Leukemia 21: 1104-1108.

5. Kondo T, Mori A, Darmanin S, Hashino S, Tanaka J, Asaka M (2008) The seventh pathogenic fusion gene FIP1L1-RARA was isolated from a t(4;17)-positive acute promyelocytic leukemia. Haematologica 93; 1414-1416.

6. Menezes J, Acquadro F, Perez-Pons de la Villa C, García-Sánchez F, Álvarez S, Cigudosa JC (2011) FIP1L1/RARA with breakpoint at FIP1L1 intron 13: a variant translocation in acute promyelocytic leukemia. Haematologica 96: 1565-1566.

7. Stover EH, Chen J, Folens C, Lee BH, Mentens N, Marynen P, Williams IR, Gilliland DG, Cools J (2006) Activation of FIP1L1-PDGFRalpha requires disruption of the juxtamembrane domain of PDGFRalpha and is FIP1L1-independent. Proc. Natl. Acad. Sci. U S A 103: 8078-8083.

8. Buitenhuis M, Verhagen LP, Cools J, Coffer PJ (2007) Molecular mechanisms underlying FIP1L1-PDGFRA-mediated myeloproliferation. Cancer Res. 67:3759-66. 
9. Toffalini F, Kallin A, Vandenberghe P, Pierre P, Michaux L, Cools J, Demoulin JB (2009) The fusion proteins TEL-PDGFRbeta and FIP1L1-PDGFRalpha escape ubiquitination and degradation. Haematologica 94: 1085-1093.

10. Kondo T, Kobayashi M, Tanaka J, Yokoyama A, Suzuki S, Kato N, Onozawa M, Chiba K, Hashino S, Imamura M, Minami Y, Minamino N, Asaka M (2004) Rapid degradation of Cdt1 upon UV-induced DNA damage is mediated by SCFSkp2 complex, J. Biol. Chem. 279: 27315-27319.

11. Kaufmann I, Martin G, Friedlein A, Langen H, Keller W (2004) Human Fip1 is a subunit of CPSF that binds to U-rich RNA elements and stimulates poly(A) polymerase. EMBO J. 23: 616-626.

12. Walz C, Score J, Mix J, Cilloni D, Roche-Lestienne C, Yeh RF, Wiemels JL, Ottaviani E, Erben P, Hochhaus A, Baccarani M, Grimwade D, Preudhomme C, Apperley J, Martinelli G, Saglio G, Cross NC, Reiter A; European LeukemiaNet. (2009) The molecular anatomy of the FIP1L1-PDGFRA fusion gene. Leukemia 23: 271-278.

13. Okabe M, Uehara Y, Miyagishima T, Itaya T, Tanaka M, Kuni-Eda Y, Kurosawa M, Miyazaki T (1992) Effect of herbimycin A, an antagonist of tyrosine kinase, on bcr/abl oncoprotein-associated cell proliferations: abrogative effect on the transformation of murine hematopoietic cells by transfection of a retroviral vector expressing oncoprotein P210bcr/abl and preferential inhibition on Ph1-positive leukemia cell growth. Blood 80: 1330-1338. 
Figure Legends

Figure 1 Schematic representation of FIP1L1-RARA and FIP1L1-PDGFRA constructs

a) Schematic representation of FIP1L1-RARA deletion mutants. FIP1L1 consists of 594 amino acids (aa) and RARA consists of 478 aa. In FIP1L1-RARA (831 aa), the N-terminal portion of FIP1L1 (428 aa) is fused to the C-terminal portion of RARA (403 aa). The conserved FIP1 motif spans aa 153 to 204 in FIP1L1. FIP1L1-N152, -N242 and -N339 consist of N-terminal 152 aa, 242 aa and 339 aa respectively. FIP1L1-RARA-dN134 and -dN235 lack N-terminal 134 aa and 235 aa respectively.

b) Schematic representation of internal deletion mutants of FIP1L1-RARA. FIP1L1-RARA-dFIP1 lacks aa 153 to 212, FIP1L1-RARA-dEx9 lacks aa 213 to 235 (corresponding to exon 9), and FIP1L1-RARA-dFIP1/Ex9 lacks aa 153 to 235 of FIP1L1-RARA.

c) Schematic representation of FIP1L1-PDGFRA and its derivatives. PDGFRA consists of 1059 aa; the N-terminal portion of FIP1L1 (339 aa) is fused to the C-terminal portion of PDGFRA (480 aa) in FIP1L1-PDGFRA-FL. FIP1L1-PDGFRA-dFIP1/Ex9 lacks aa 153 to 235 of FIP1L1-PDGFRA and PDGFRA-C lacks the FIP1L1 portion.

Figure 2. The FIP1 motif is crucial for homodimer formation and transcriptional repressor activity of FIP1L1-RARA.

a) The FIP1L1 region comprising aa 135 to 236 is crucial for FIP1L1-RARA homodimerization. HEK293 cells were transfected with pCGT-FIP1L1-RARA together with a control vector, pFLAG-FIP1L1-RARA, pFLAG-FIP1L1-N152, pFLAG-FIP1L1-N242, pFLAG-FIP1L1-N339, pFLAG-FIP1L1-RARA-dN134 or pFLAG-FIP1L1-RARA-d235 respectively (lanes 2 to 8). Lane 1 was loaded with untransfected HEK293 cell lysate. T7-FIP1L1-RARA expression levels were examined by immunoblotting with an anti-T7 antibody (upper panel). The association between T7-FIP1L1-RARA and FLAG-tagged derivatives was analyzed by immunoprecipitation with an 
anti-FLAG M2 antibody and immunoblotting with anti-T7 (middle panel) and anti-FLAG M2 (lower panel) antibodies. The asterisk indicates the immunoglobulin heavy chain.

b) The FIP1 motif plays a pivotal role in FIP1L1-RARA homodimerization. HEK293 cells were transfected with pCGT-FIP1L1-RARA, together with a control vector, $p F L A G-F I P 1 L 1-R A R A, ~ p F L A G-F I P 1 L 1-R A R A-d F I P 1$, pFLAG-FIP1L1-RARA-dEx9 or pFLAG-FIP1L1-RARA-dFIP1/Ex9 respectively (lanes 2 to 6). Lane 1 was loaded with untransfected HEK293 cell lysate. The expression levels of T7-FIP1L1-RARA and FLAG-FIP1L1-RARA were examined by immunoblotting with anti-T7 and anti-FLAG M2 antibodies (WCL), and the association between T7-FIP1L1-RARA and FLAG-tagged derivatives was analyzed by immunoprecipitation with anti-FLAG M2 antibody and immunoblotting with anti-T7 and anti-FLAG M2 antibodies (IP).

c) HEK293 cells were transfected, in $35-\mathrm{mm}$ dishes, with $0.25 \mu \mathrm{g}$ retinoic acid responsive-luciferase vector, which contains seven repeats of the retinoic acid-response element (RARE) in the RAR62 gene, combined with $2 \mu \mathrm{g}$ empty vector or the expression vectors pFLAG-FIP1L1-RARA, pFLAG-FIP1L1-RARA-dFIP1, pFLAG-FIP1L1-RARA-dEx9 or pFLAG-FIP1L1-RARA-dFIP1/Ex9 respectively. One day after transfection, the culture media were exchanged with fresh culture media supplemented with the indicated concentration of ATRA. Following two days of 10nM ATRA treatment, the cells were harvested and luciferase activities were analyzed. The luciferase activity without ATRA treatment was arbitrarily assigned a value of 1.0 and the results are shown as the mean \pm SD. pFLAG-FIP1L1-RARA and pFLAG-FIP1L1-RARA-dEx9 significantly repressed retinoic acid-dependent transcriptional activity ( $<<0.04$, Mann-Whitney test). The analysis was performed in triplicate assays and the results were reproducible.

Figure 3. FIP1L1-PDGFRA homodimerization is not mediated by the FIP1 motif. 
a) FIP1L1-PDGFRA homodimerization is independent of the FIP1 motif. FLAG-tagged or T7-tagged FIP1L1-PDGFRA-FL and-dFIP1/Ex9 were expressed in HEK293 cells and their reciprocal association was examined. The association between the T7-tagged molecule and the FLAG-tagged molecule was analyzed by immunoprecipitation with anti-T7 antibody and immunoblotting with anti-FLAG M2 antibody.

b) PDGFRA-C, by itself, has the ability to form a homodimer. FLAG-tagged or T7-tagged FIP1L1-PDGFRA-FL and PDFGRA-C were expressed in HEK293 cells and their reciprocal association was examined. The association between the FLAG-tagged molecule and the T7-tagged molecule was analyzed by immunoprecipitation with anti-T7 antibody and by immunoblotting with anti-FLAG M2 antibody.

c) Both FIP1L1-PDGFRA and PDGFRA-C are kinase-active. FLAG-tagged FIP1L1-PDGFRA-FL, FIP1L1-PDGFRA-dFIP1/Ex9, PDFGRA-C or FIP1L1-PDGFRA-KD were individually expressed in HEK293 cells and the status of tyrosine phosphorylation was examined. After immunoprecipitaion with anti-FLAG M2 antibody, the expression level of FLAG-tagged molecules was examined with anti-FLAG M2 antibody, and the phosphorylation of tyrosine residues was analyzed with an anti-phosphotyrosine antibody (PY20).

Figure 4. Growth characteristics of BAF-B03-derived stable transformants expressing T7-tagged FIP1L1-PDGFRA-FL, FIP1L1-PDGFRA-dFIP1/Ex9 or PDFGRA-C.

a) BAF-B03-derived stable transformants expressing T7-tagged FIP1L1-PDGFRA-FL, FIP1L1-PDGFRA-dFIP1/Ex9 or PDFGRA-C. The expression level of each molecule was examined by immunoblotting (lane 1: empty vector, lane 2: T7- FIP1L1-PDGFRA-FL, lane 3: T7-FIP1L1-PDGFRA-dFIP1/Ex9, lane 4: T7-PDFGRA-C).

b) $\sim$ e) Each cell line was cultured with or without murine IL-3, and the cell number was counted daily until day 4 . The results are shown as the mean \pm SD. Closed triangles indicate growth in the presence of IL-3 and 
closed circles indicate growth in the absence of IL-3. The analysis was performed in triplicate assays and the results were reproducible.

Figure 5 FIP1L1-PDFRA locates primarily in the nucleus.

a) d) T7-tagged FIP1L1-PDFRA-FL(Figure 5b), T7-tagged FIP1L1-PDFRA-dFIP1/Ex9 (Figure 5c) and T7-tagged PDGFRA-C (Figure 5d) were expressed in 293 cells and immunostained with anti-T7 antibody. In Figure 5a, an empty vector was used as a negative control. The nucleus was simultaneously visualized by $4^{\prime}, 6^{\prime}$ -diamidine-2-phenylindole-dihydrochloride (DAPI). 


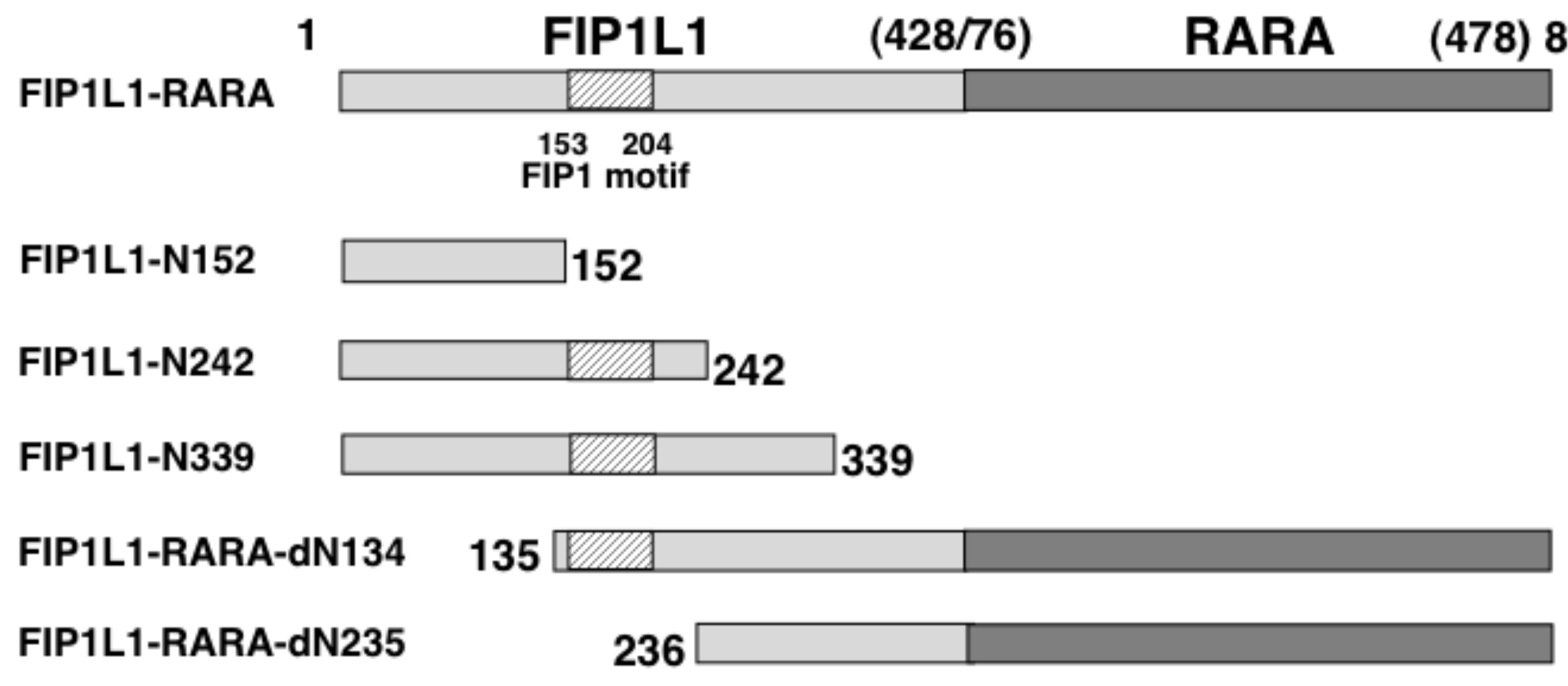

Figure 1a 


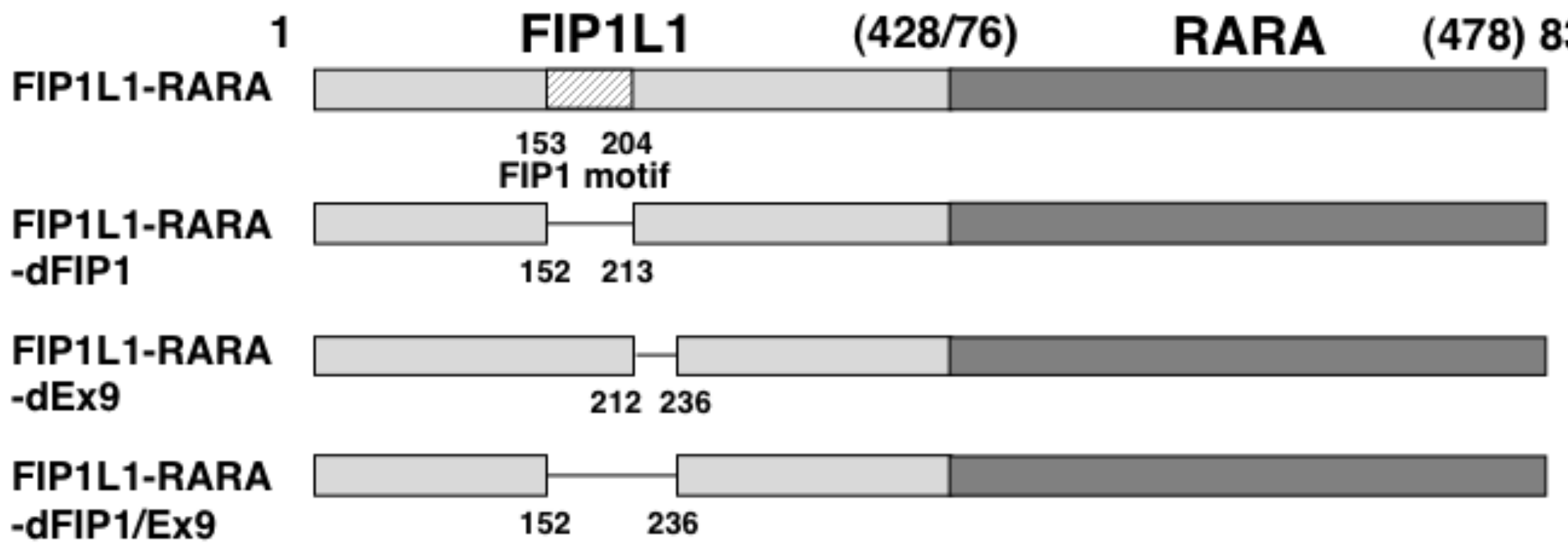

Figure 1b 


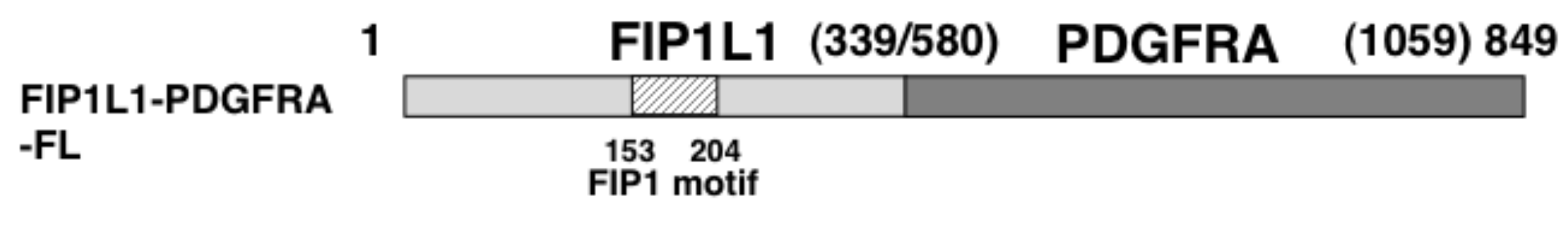

FIP1L1-PDGFRA -dFIP1/Ex9

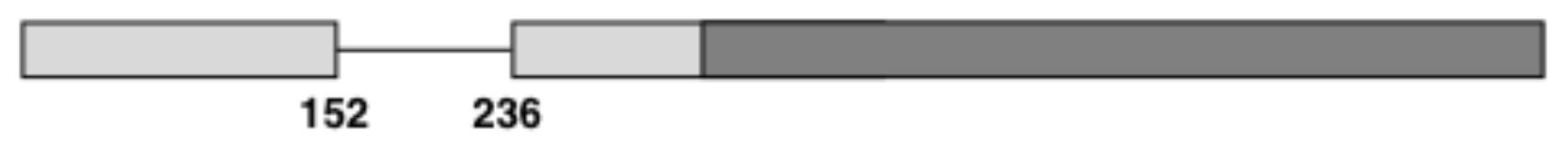

340

849

PDGFRA-C 

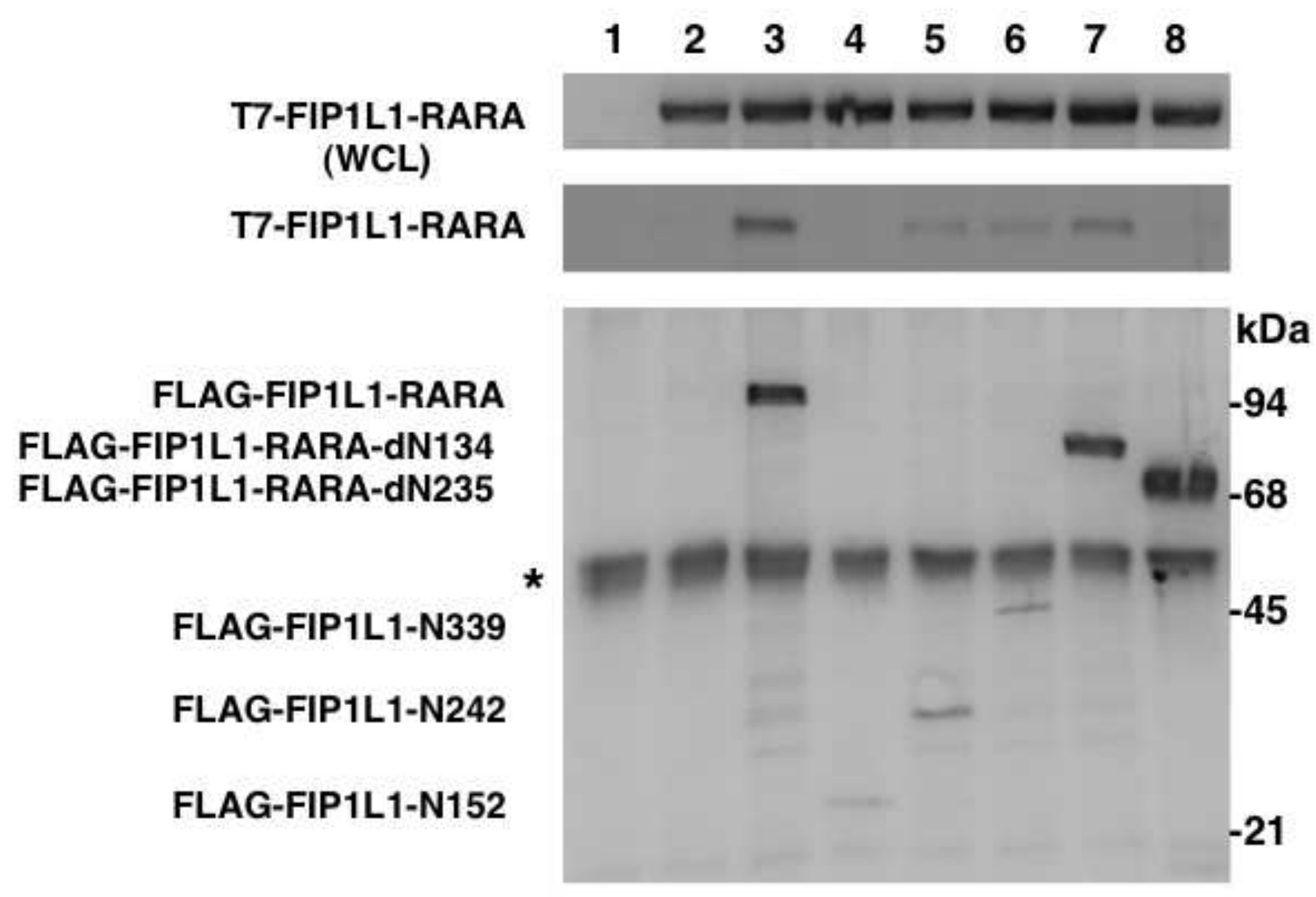

Figure 2a 


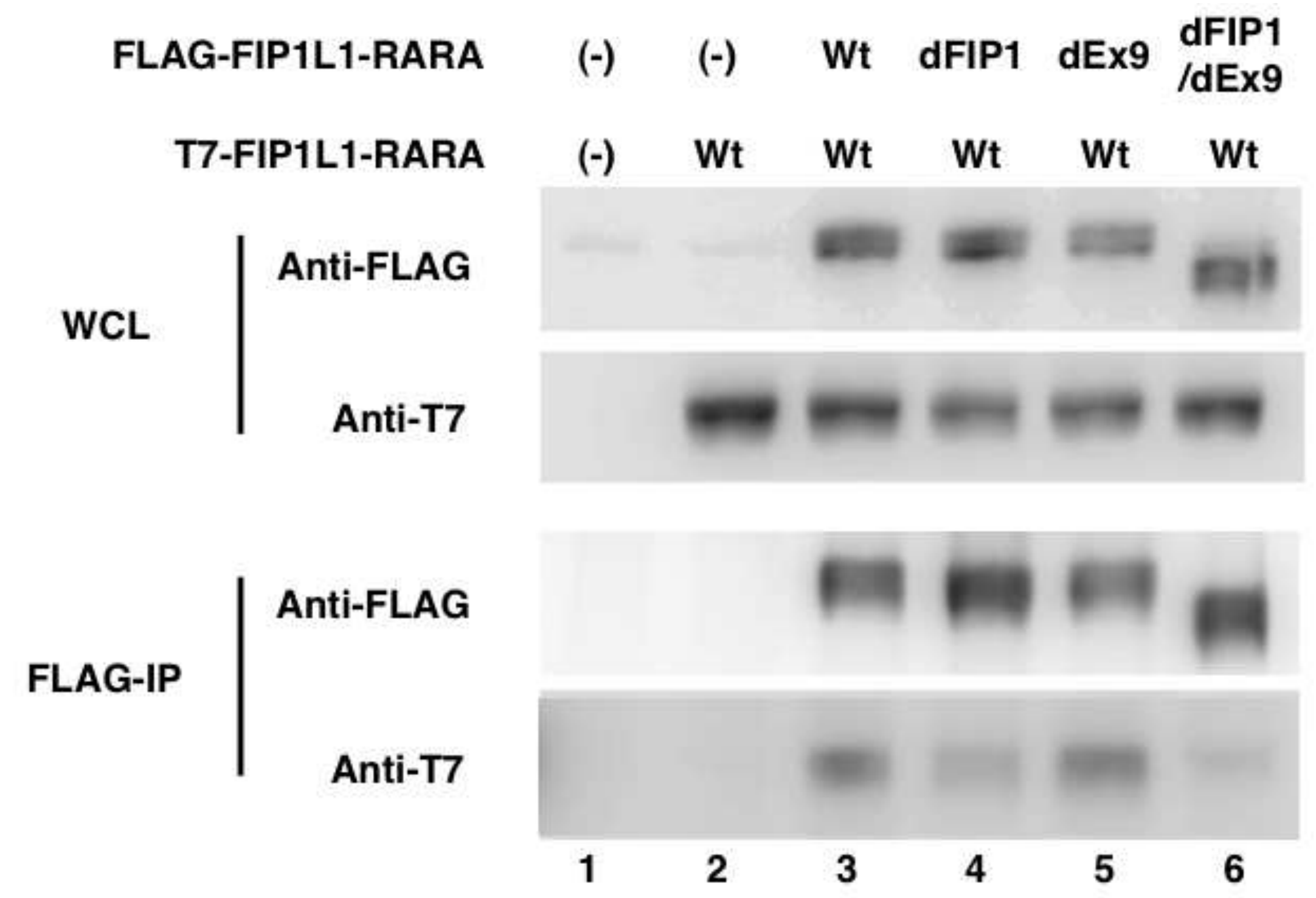

Figure 2b 


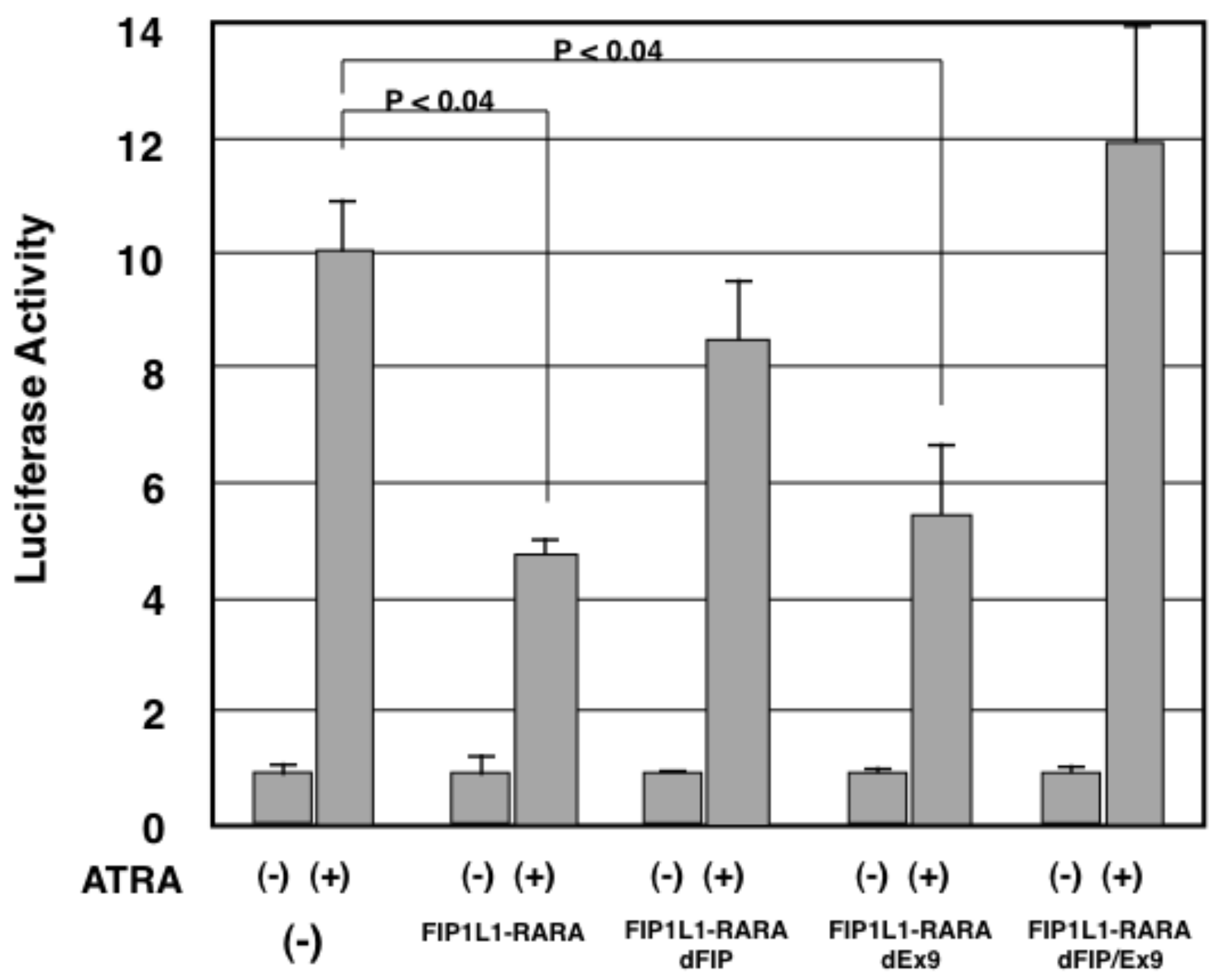

Figure 2c 

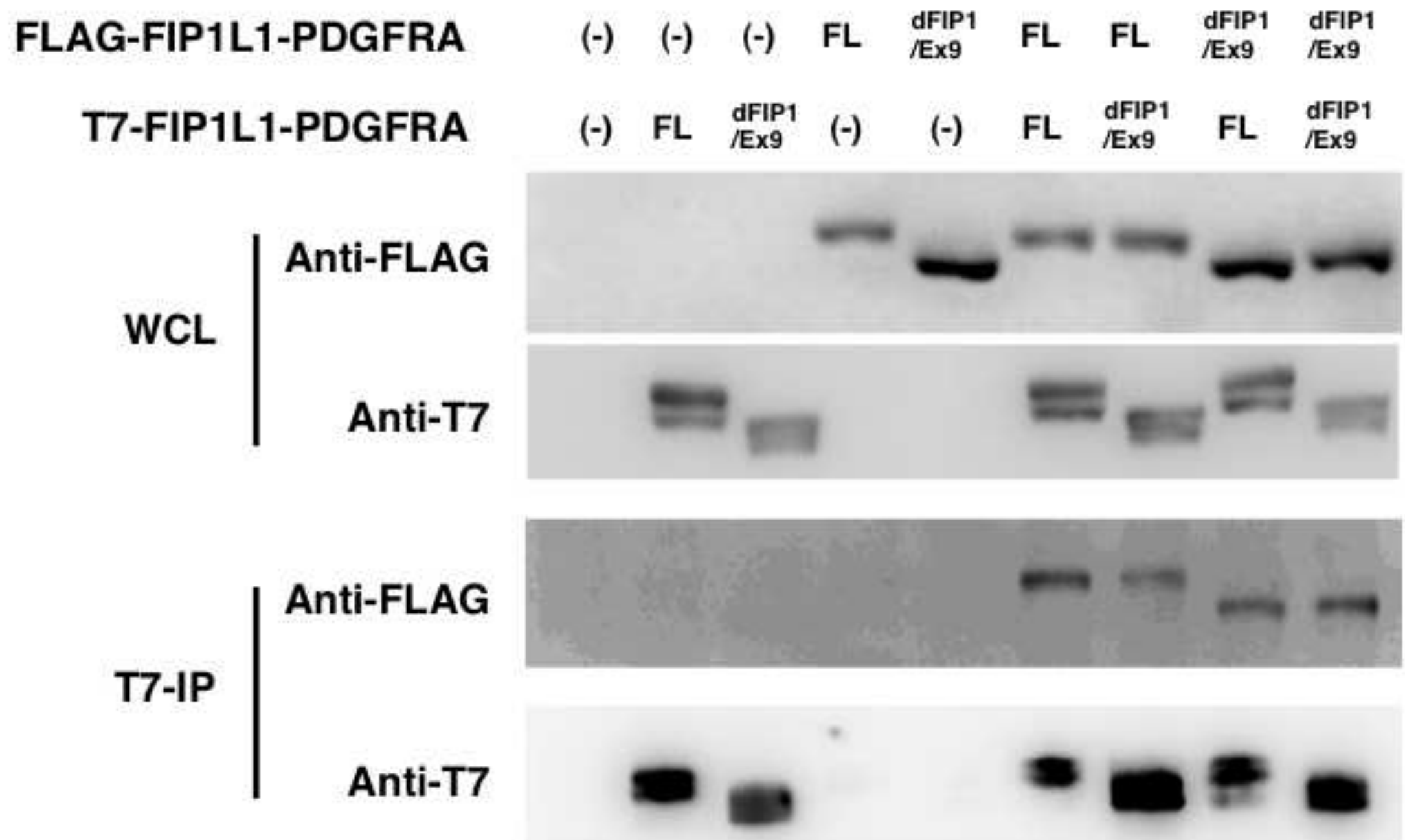

$\begin{array}{lllllllll}1 & 2 & 3 & 4 & 5 & 6 & 7 & 8 & 9\end{array}$

Figure 3a 


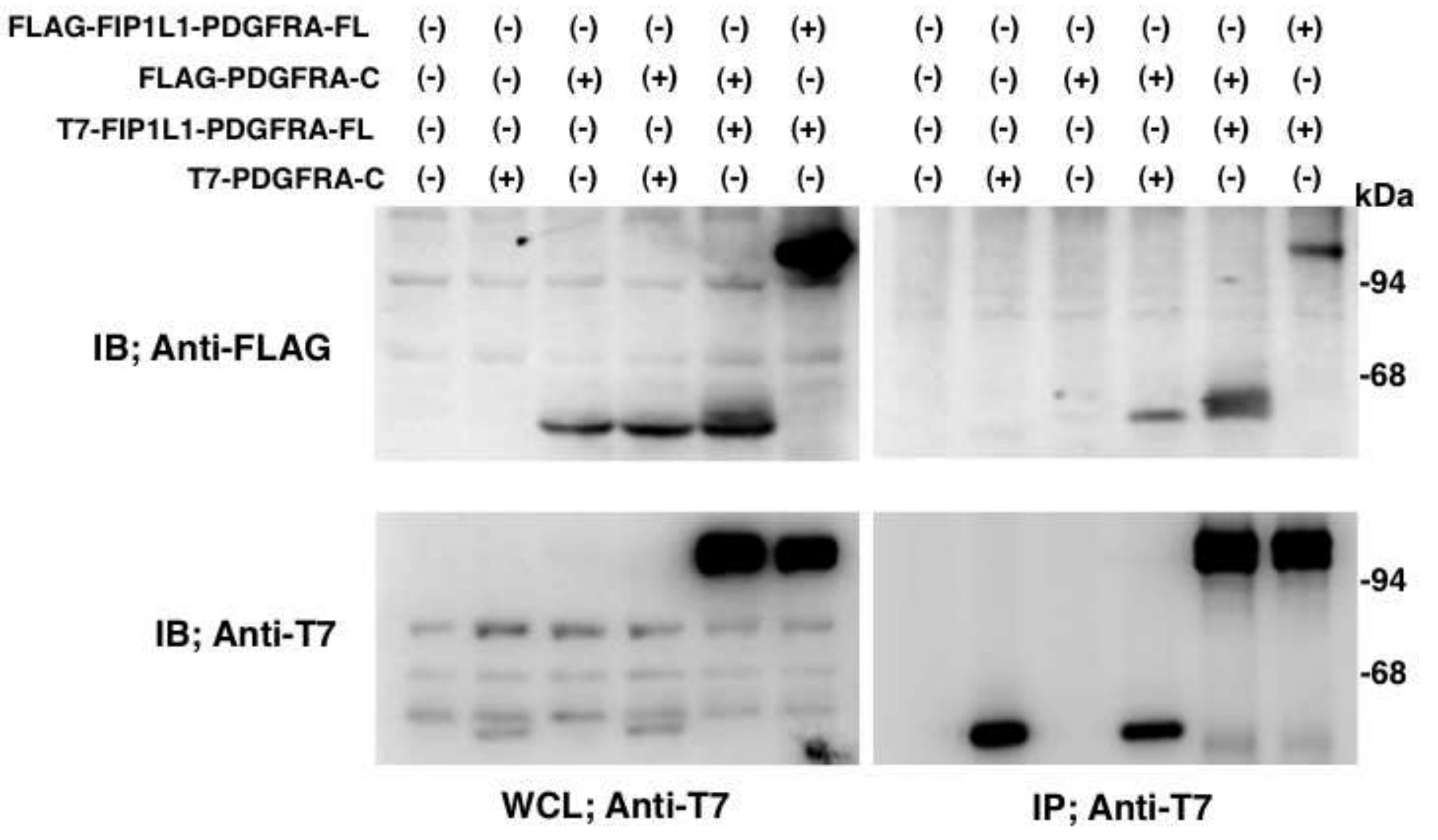

Figure $3 b$ 


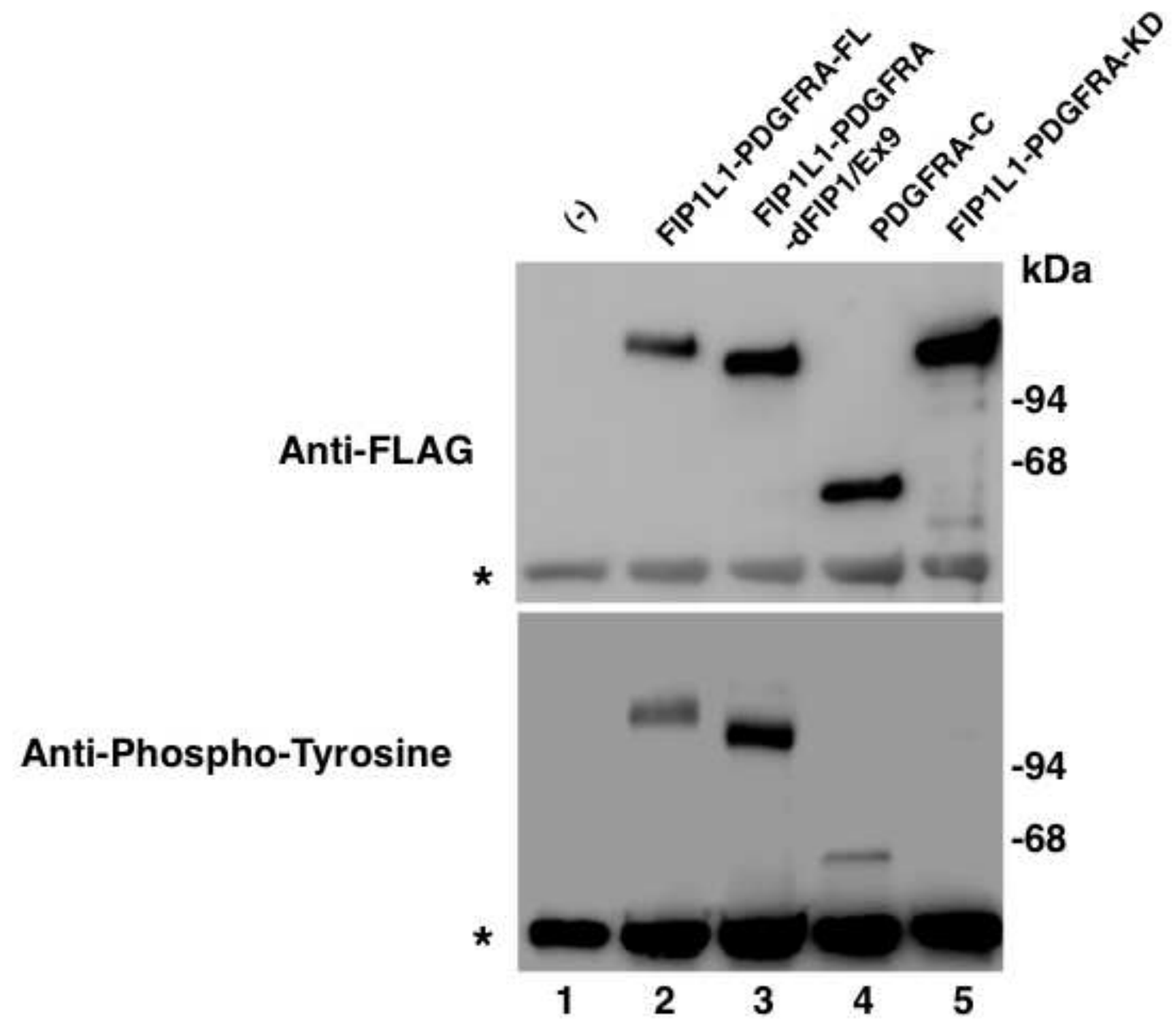

Figure 3c 


\section{Anti-T7}

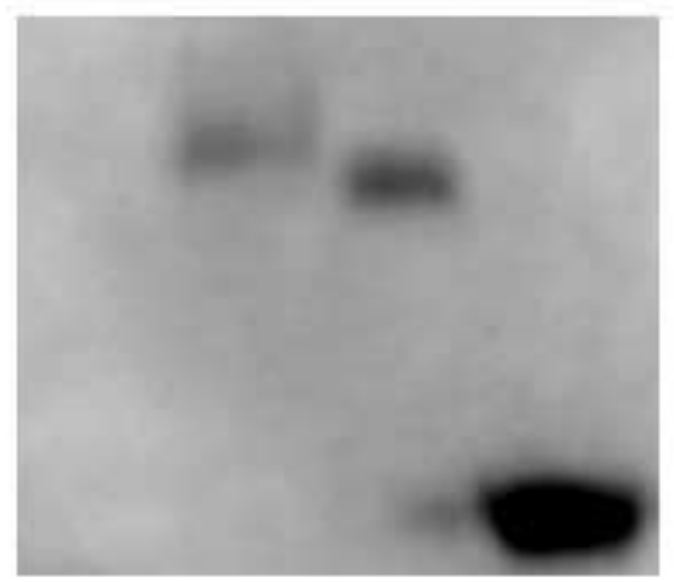

\section{Anti-Actin}

\section{$\begin{array}{llll}1 & 2 & 3 & 4\end{array}$}

Figure 4a

Click here to download high resolution imag 
(X10 ${ }^{3}$ cells)

\section{BAF-B03/Empty}

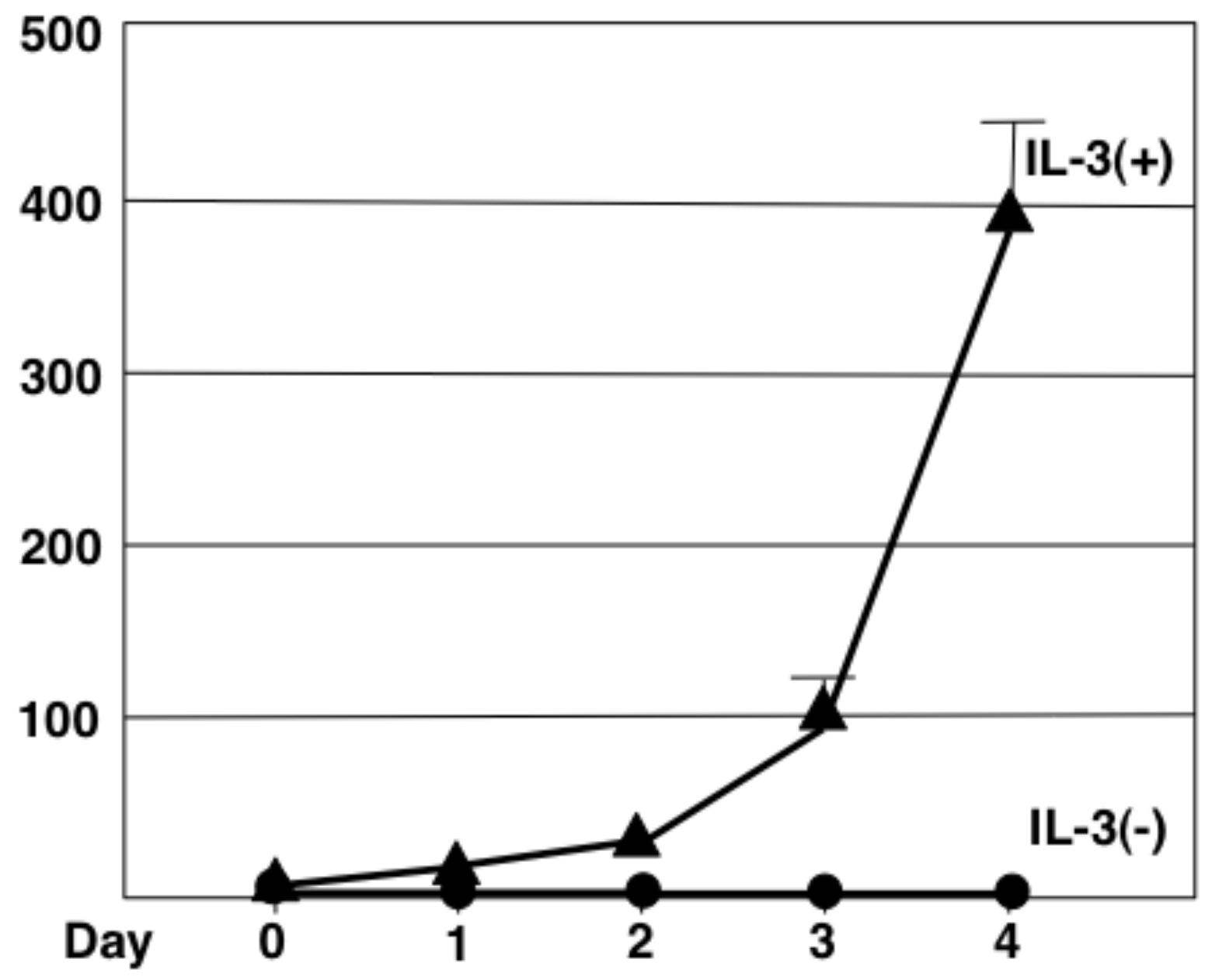

Figure 4b 
$\left(\mathrm{X} 10^{3}\right.$ cells)

BAF-B03/FP-FL

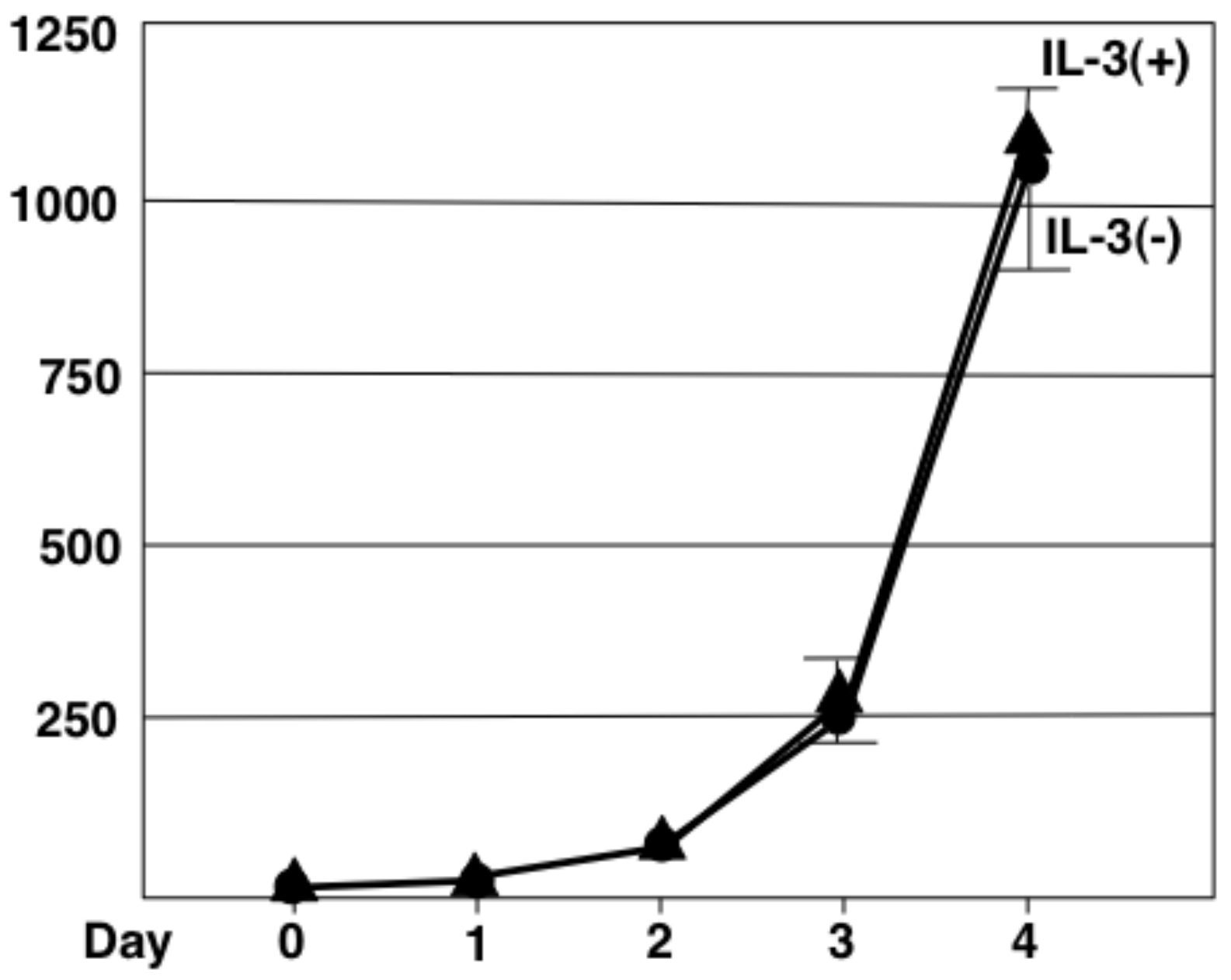

Figure 4c 
(X10 $0^{3}$ cells)

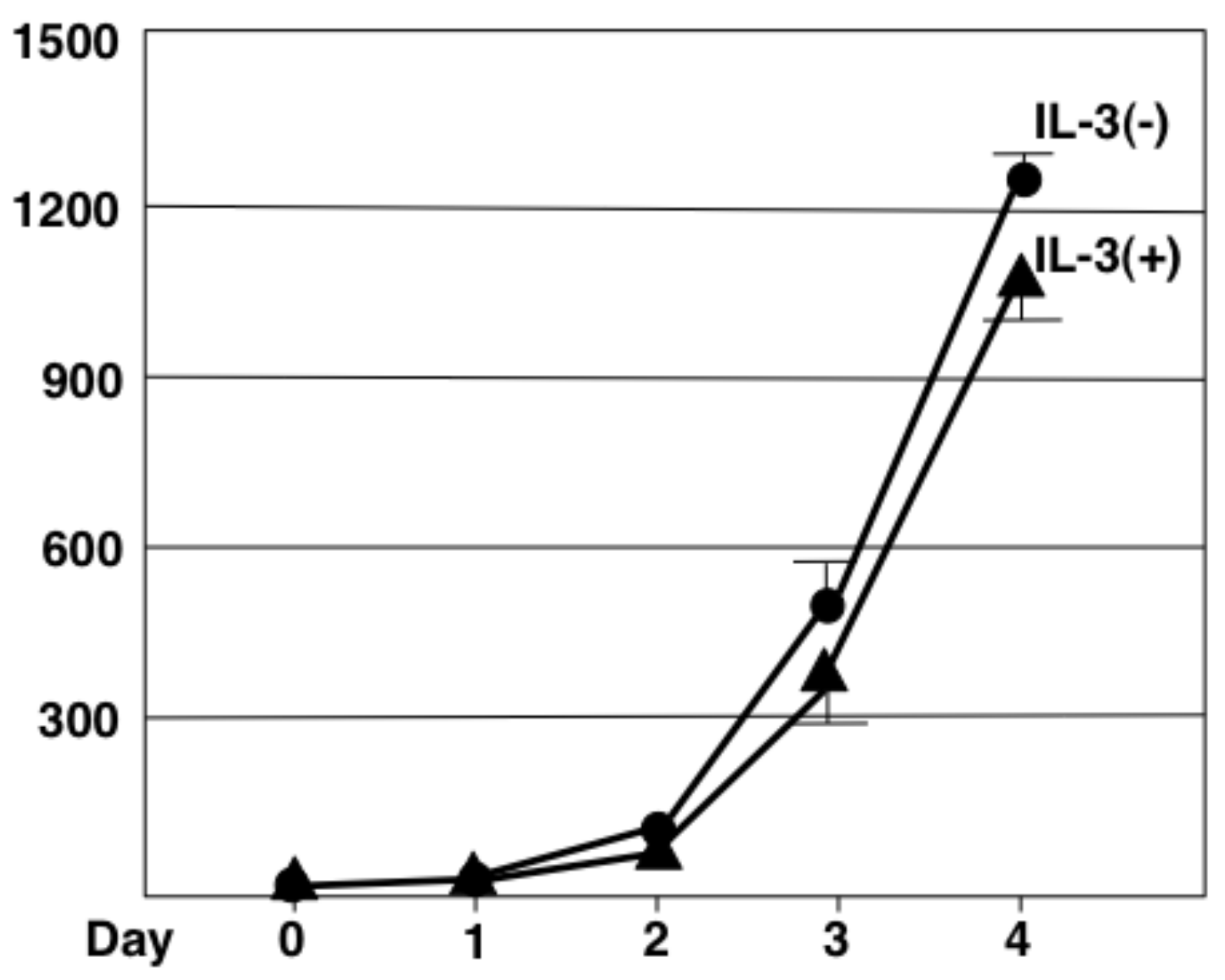

BAF-B03/FP-dFIP/Ex9
Figure 4d 
(X10 $0^{3}$ cells)

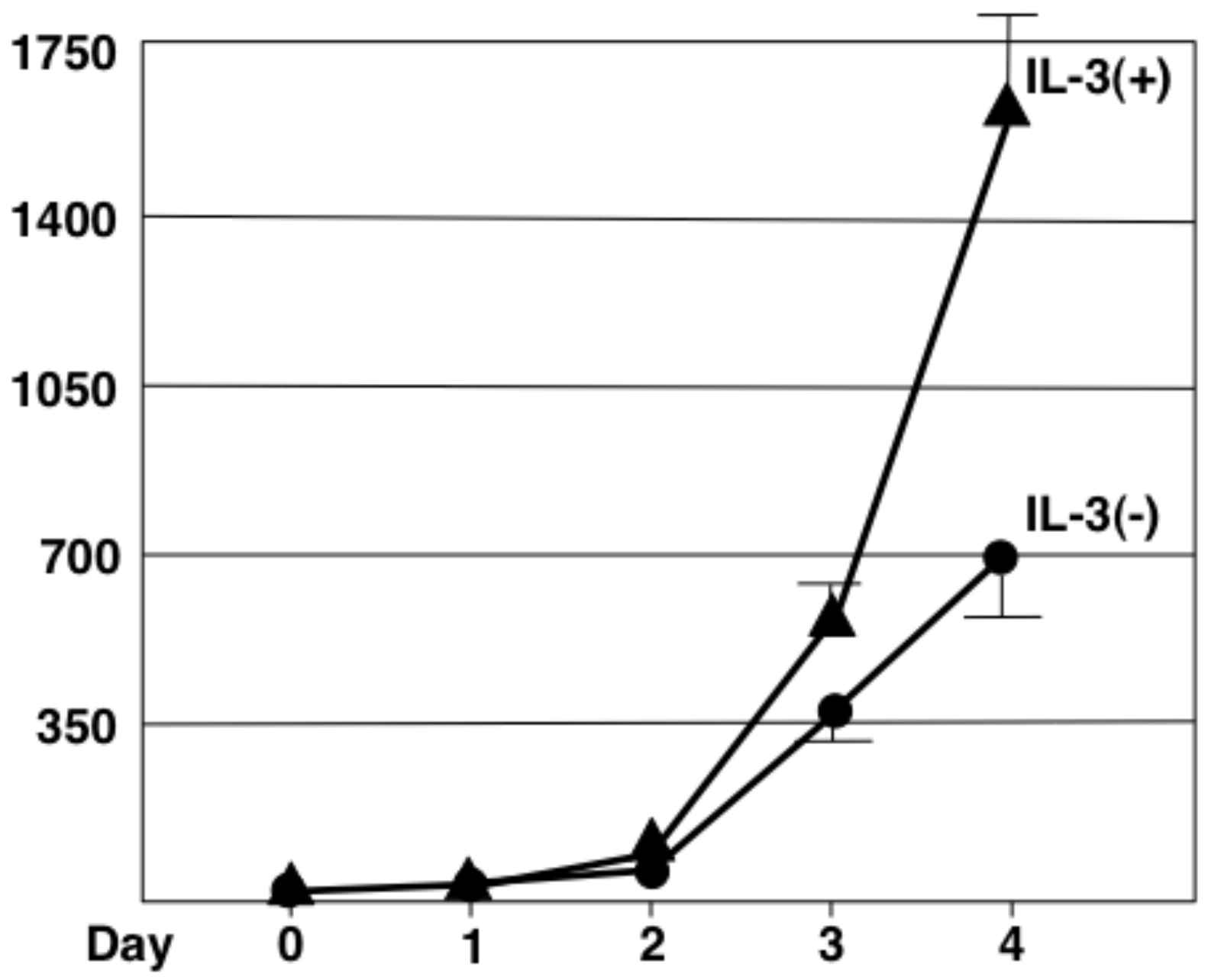

Figure 4e 


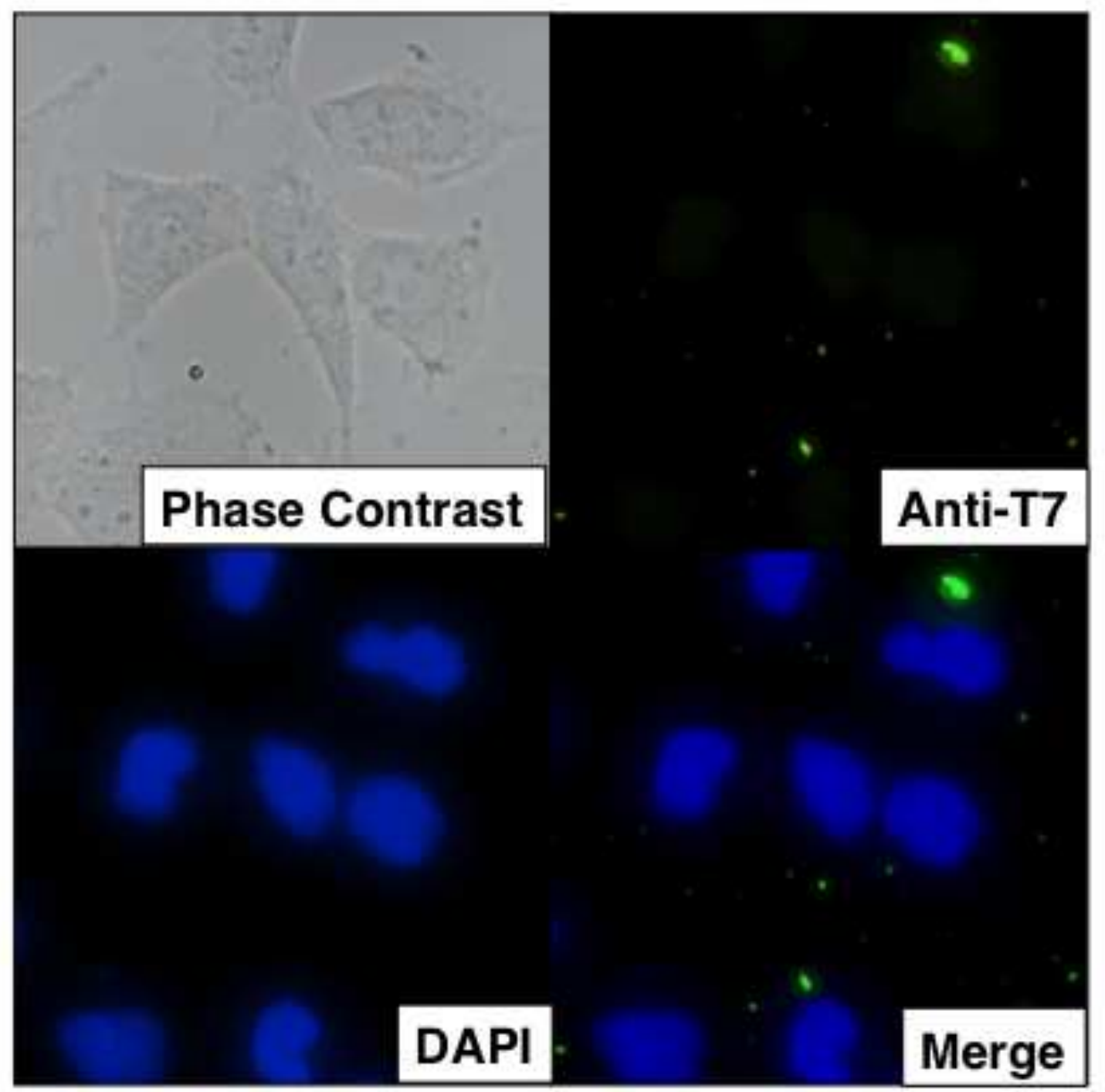

Figure 5a

\section{Phase Contrast}

Merge 


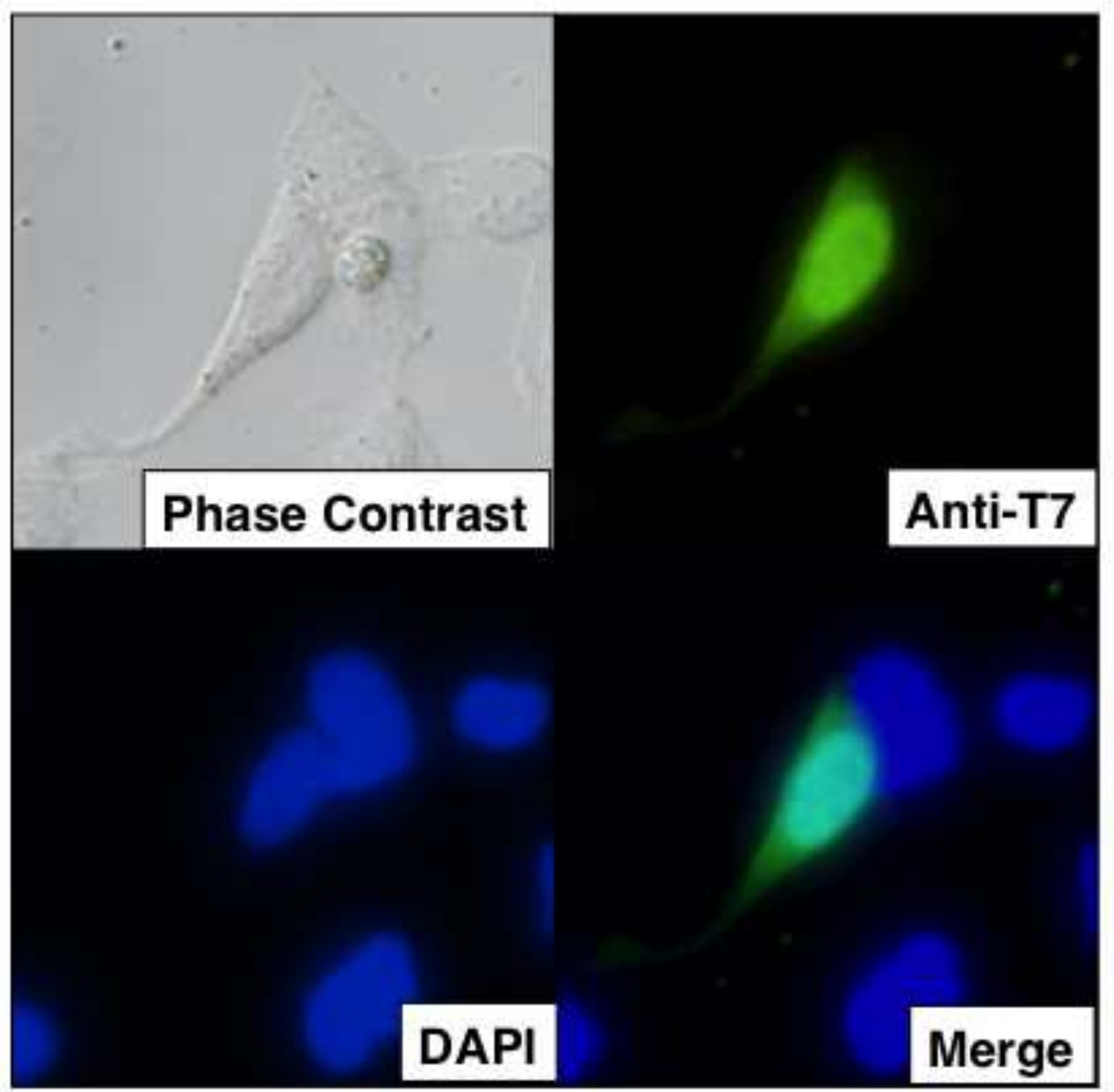

Figure 5b 


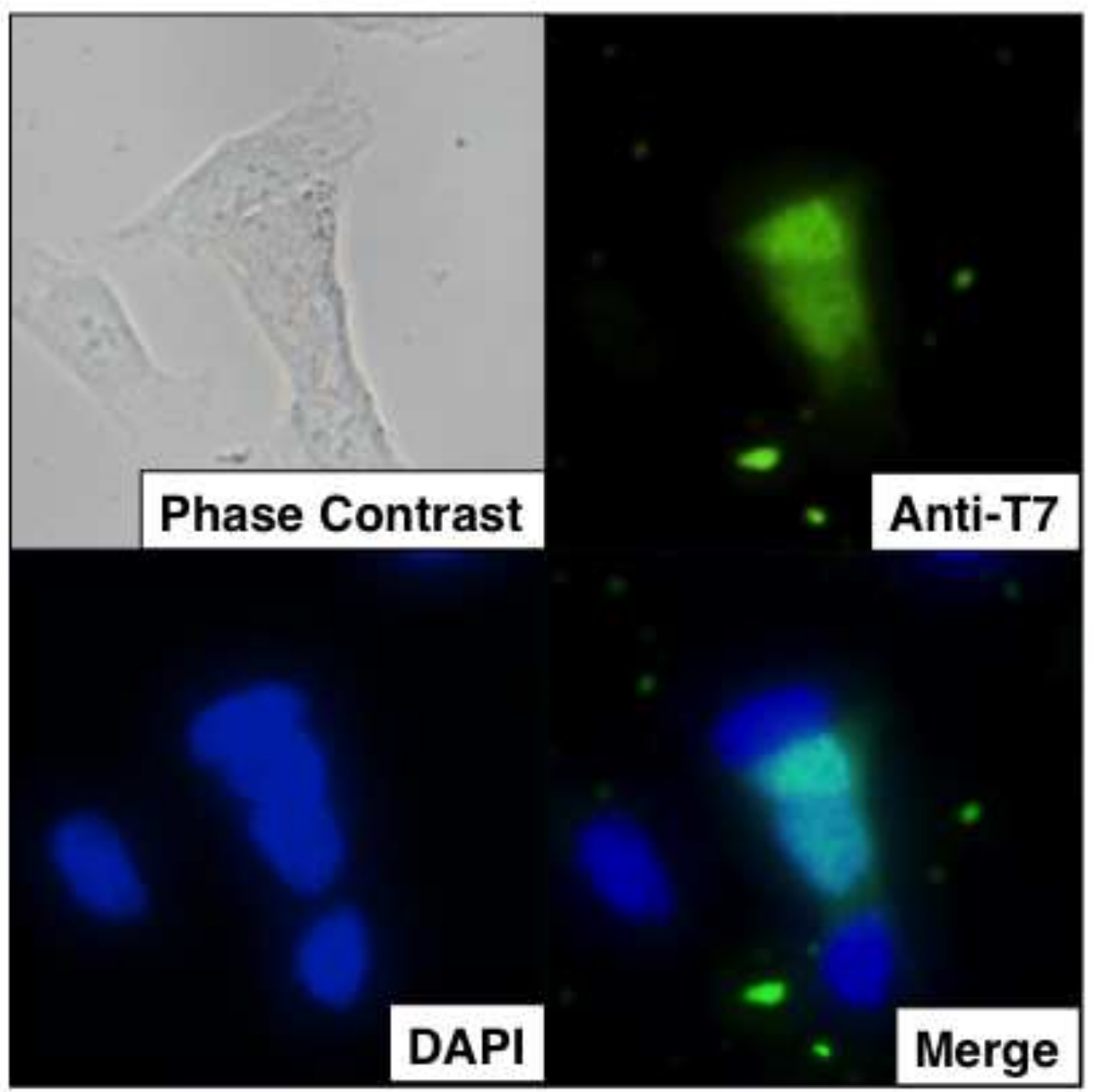

Figure 5c

\section{Phase Contrast}

Merge 


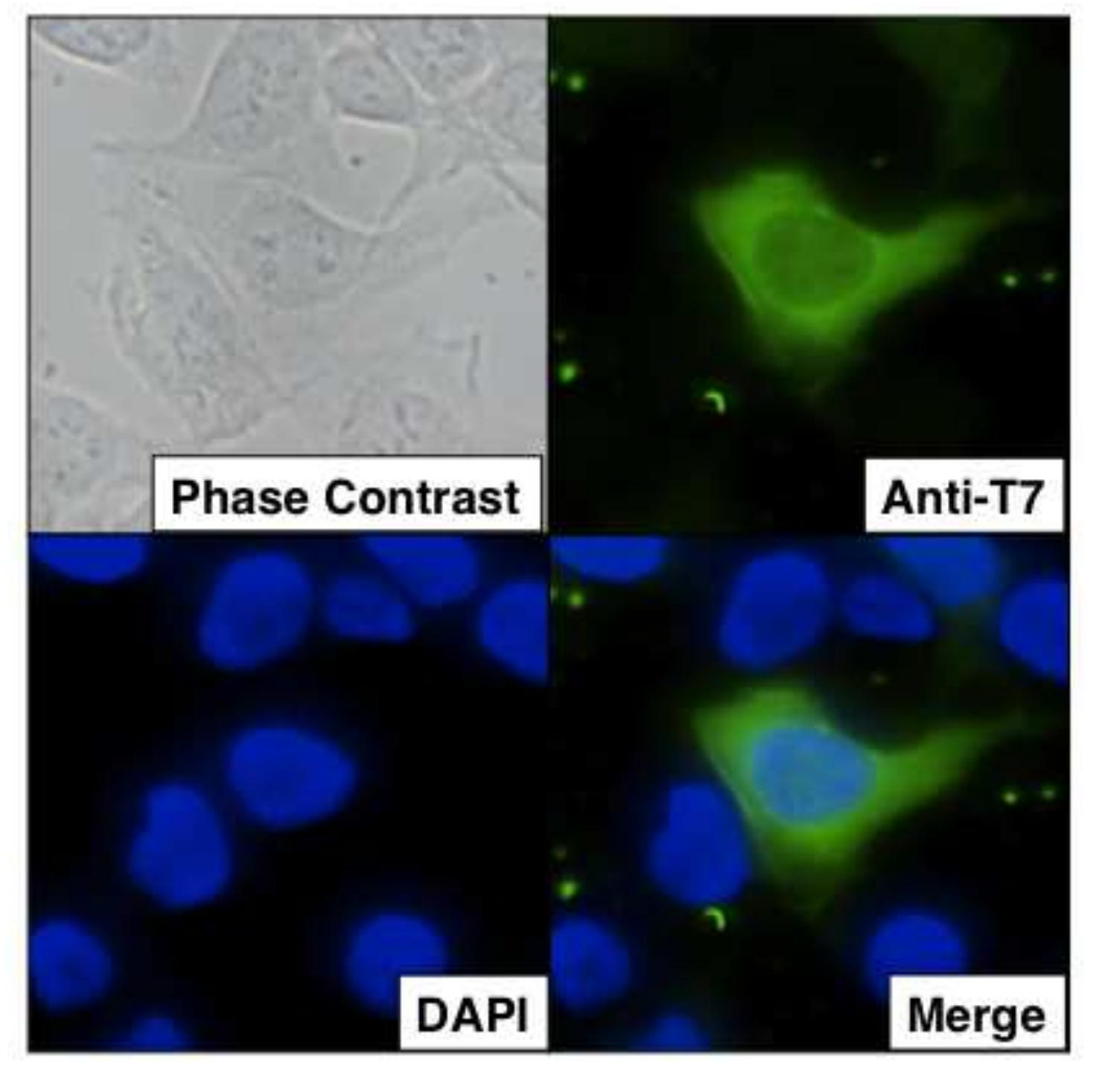

Figure 5d

\section{Phase Contrast}

Merge

DAPI

Anti-T7 Figure 5d

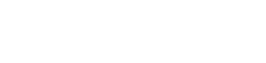

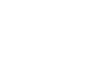

